\title{
Circulating Adipocytokines and Chronic Kidney Disease
}

\author{
Katherine T. Mills' ${ }^{1}$, L. Lee Hamm ${ }^{2,3}$, A. Brent Alper ${ }^{2,3}$, Chad Miller ${ }^{2}$, Alhakam Hudaihed ${ }^{2}$, \\ Saravanan Balamuthusamy ${ }^{2}$, Chung-Shiuan Chen ${ }^{1}$, Yanxi Liu ${ }^{1}$, Joseph Tarsia ${ }^{2}$, Nader Rifai ${ }^{4,5}$, \\ Myra Kleinpeter ${ }^{2,3}$, Jiang $\mathrm{He}^{1,2}$, Jing Chen ${ }^{1,2,3 *}$
}

1 Department of Epidemiology, Tulane University School of Public Health and Tropical Medicine, New Orleans, Louisiana, United States of America, 2 Department of Medicine, Tulane University School of Medicine, New Orleans, Louisiana, United States of America, $\mathbf{3}$ Tulane University Hypertension and Renal Center of Excellence, Tulane University School of Medicine, New Orleans, Louisiana, United States of America, 4 Department of Laboratory Medicine, Children' Hospital, Boston, Massachusetts, United States of America, 5 King Abdulaziz University, Jeddah, Saudi Arabia

\begin{abstract}
Background: Adipokines have been associated with atherosclerotic heart disease, which shares many common risk factors with chronic kidney disease (CKD), but their relationship with CKD has not been well characterized.

Methods: We investigated the association of plasma leptin, resistin and adiponectin with CKD in 201 patients with CKD and 201 controls without. CKD was defined as estimated glomerular filtration rate (eGFR) $<60 \mathrm{~mL} / \mathrm{min} / 1.73 \mathrm{~m}^{2}$ or presence of albuminuria. Quantile regression and logistic regression models were used to examine the association between adipokines and CKD adjusting for multiple confounding factors.

Results: Compared to controls, adjusted median leptin (38.2 vs. $17.2 \mathrm{ng} / \mathrm{mL}, \mathrm{p}<0.0001$ ) and adjusted mean resistin (16.2 vs $9.0 \mathrm{ng} / \mathrm{mL}, \mathrm{p}<0.0001)$ were significantly higher in CKD cases. The multiple-adjusted odds ratio (95\% confidence interval) of CKD comparing the highest tertile to the lower two tertiles was $2.3(1.1,4.9)$ for leptin and $12.7(6.5,24.6)$ for resistin. Median adiponectin was not significantly different in cases and controls, but the odds ratio comparing the highest tertile to the lower two tertiles was significant $(1.9 ; 95 \% \mathrm{Cl}, 1.1,3.6)$. In addition, higher leptin, resistin, and adiponectin were independently associated with lower eGFR and higher urinary albumin levels.

Conclusions: These findings suggest that adipocytokines are independently and significantly associated with the risk and severity of CKD. Longitudinal studies are warranted to evaluate the prospective relationship of adipocytokines to the development and progression of CKD.
\end{abstract}

Citation: Mills KT, Hamm LL, Alper AB, Miller C, Hudaihed A, et al. (2013) Circulating Adipocytokines and Chronic Kidney Disease. PLoS ONE 8(10): e76902. doi:10.1371/journal.pone.0076902

Editor: Cordula M. Stover, University of Leicester, United Kingdom

Received May 31, 2013; Accepted August 28, 2013; Published October 7, 2013

Copyright: $\odot 2013$ Mills et al. This is an open-access article distributed under the terms of the Creative Commons Attribution License, which permits unrestricted use, distribution, and reproduction in any medium, provided the original author and source are credited.

Funding: This study was supported by grants (P20-RR017659 and P30-GM103337) from the National Center for Research Resources, National Institutes of Health, Bethesda, Maryland (http://www.nih.gov/about/almanac/organization/NCRR.htm) and partially supported by a Clinical and Translational Clinical Research Cente grant from the Louisiana Board of Regents RC/EEP Fund (http://web.laregents.org/programs/rceep/). The funders had no role in study design, data collection and analysis, decision to publish, or preparation of the manuscript.

Competing Interests: The authors have declared that no competing interests exist.

*E-mail: jchen@tulane.edu

\section{Introduction}

The prevalence of chronic kidney disease (CKD) is high and increasing in the US general population [1,2]. CKD is associated with an increased risk of end-stage kidney disease (ESKD), cardiovascular disease (GVD), and premature death [3-5. CKD and CVD are closely associated with each other and share many common risk factors [6,7]. Recent research has revealed that adipokines are associated with atherosclerotic CVD [8], but their associations with CKD have not been well characterized.

An elevated level of leptin has been found in patients with ESKD $[9,10]$, but this association is inconsistent among patients with pre-dialysis CKD: an elevated level in CKD patients [11-14] or no difference [15] have been reported. Previous studies have also reported a higher level of resistin among patients with stage 3 and 4 CKD [16-18]. In addition, higher adiponectin levels in those with CKD [19] and ESKD [20] or no difference in adiponectin levels between individuals with and without CKD have been reported [13,17,21].

Much of the previous work on the association between leptin, resistin, and adiponectin and CKD has produced conflicting results and has originated primarily from studies with small sample sizes and/or unadjusted comparisons between CKD patients and those without CKD. Our objective is to investigate the association of plasma leptin, resistin, and adiponectin with CKD in a large case-control study after adjusting for multiple important risk factors for CKD. Additionally, we will examine the relationship between plasma leptin, resistin, and total adiponectin and severity of CKD, measured by estimated glomerular filtration rate (eGFR) and urinary albumin. 


\section{Subjects and Methods}

\section{Ethics statement}

Tulane University Internal Review Board approved this study, and written informed consent was obtained at the screening visit from all study participants. This study adheres to the Declaration of Helsinki.

\section{Study participants}

We recruited 201 CKD patients and 201 controls without CKD in the greater New Orleans, Louisiana area from 2007 to 2010. CKD patients aged 21-74 years were identified from nephrology and internal medicine clinics in the study area. CKD was defined as estimated glomerular filtration rate (eGFR) $<60 \mathrm{ml} / \mathrm{min} /$ $1.73 \mathrm{~m}^{2}$ or presence of albuminuria ( $\geq 30 \mathrm{mg} / 24$-hours). Patients were excluded if they had a history of chronic dialysis, kidney transplants, uremia, autoimmune or chronic systemic inflammatory diseases, immunotherapy in the past six months, chemotherapy within the past two years, and current clinical trial participation that may have an impact on CKD. Additional exclusion criteria for both cases and controls were history of HIV or AIDS and inability or unwillingness to give informed consent. Controls, defined as having no evidence of CKD (eGFR $>60 \mathrm{ml} /$ $\mathrm{min} / 1.73 \mathrm{~m}^{2}$ and no persistent albuminuria), were recruited through mass mailing to residents aged $21-74$ years living in the same area.

\section{Measurements}

A standard questionnaire was administered by trained staff at a clinical visit to obtain demographic information, lifestyle risk factors (including cigarette smoking, alcohol drinking, and physical activity), self-reported history of CVD, diabetes, hypercholesterolemia, and hypertension, as well as medication use.

Three blood pressure (BP) measurements were obtained at a clinical visit by trained and certified staff using a standard mercury sphygmomanometer according to a common protocol adapted from procedures recommended by the American Heart Association [22]. Body height and weight were obtained by trained staff and used to calculate body mass index $(\mathrm{BMI}=$ weight in $\mathrm{kg} /$ height $^{2}$ in $\left.\mathrm{m}\right)$.

An overnight fasting blood sample was collected to measure plasma leptin, resistin, adiponectin, and glucose, serum creatinine and cholesterol, and triglycerides. eGFR was estimated from serum creatinine $(\mathrm{SCr})$, sex, age, and race using the CKD-Epi equation: $\mathrm{eGFR}=141 \times \min (\mathrm{SCr} / \kappa, 1)^{\alpha} \times \max (\mathrm{SCr} / \kappa, 1)^{-1.209}$ $\times 0.993^{\mathrm{Age}} \times 1.018$ (if female) $\times 1.159$ (if black), where $\kappa$ is 0.7 for females and 0.9 for males and $\alpha$ is -0.329 and -0.411 , respectively [23]. A 24-hour urinary sample was collected to measure albumin.

Serum cholesterol and triglyceride levels were assayed using an enzymatic procedure on the Hitachi 902 automatic analyzer (Roche Diagnostics, Indianapolis, IN, USA). Serum glucose was measured using a hexokinase enzymatic method (Roche Diagnostics, Indianapolis, IN, USA). Serum creatinine was measured using the Roche enzymatic method (Roche-Hitachi P-Module instrument with Roche Creatininase Plus assay, Hoffman-La Roche, Basel, Switzerland). Urinary concentrations of albumin were measured with a DCA 2000 Analyzer (Bayer AG, Leverkusen, Germany). Plasma leptin and resistin were measured using an enzymatically amplified "two-step" sandwich-type immunoassay (R\&D Systems, Minneapolis, MN, USA), and plasma adiponectin was measured using a quantitative sandwich enzyme immunoassay technique (ALPCO Diagnostics Inc., Salem, NH).

\section{Statistical analysis}

Medians and interquartile ranges (IQR) for leptin $(\mathrm{ng} / \mathrm{mL})$ and total adiponectin $(\mu \mathrm{g} / \mathrm{mL})$ were calculated for CKD patients and controls, and the Mann-Whitney test was used to test differences in the unadjusted medians. Quantile regression was used to obtain adjusted medians and IQR, and the Wald test was used to test differences in the adjusted medians between CKD patients and controls. These analyses were adjusted for age, gender, race, highschool education, current cigarette smoking, weekly alcohol consumption, regular physical activity $(\geq$ moderate or heavy activities twice per week), BMI, low-density cholesterol (LDL) cholesterol, systolic blood pressure, fasting plasma glucose, and history of cardiovascular disease. All analyses were also conducted adjusting from waist circumference instead of BMI to assess if a particular measure of adiposity has an impact on the results. Means and 95\% confidence intervals (CI) were calculated for resistin $(\mathrm{ng} / \mathrm{mL})$, a student t-test was used to test for differences in unadjusted means, and ANOVA was used for adjusted means.

Multivariate logistic regression was used to obtain adjusted odds ratios comparing the highest tertile of leptin, resistin, and total adiponectin to the lower two tertiles between CKD patients and controls. Tertiles were defined based upon measurements in the control group [24]. Multivariable linear regression was used to examine the association of eGFR and urinary albumin with leptin, resistin, and total adiponectin, after adjustment for the previously mentioned covariates. Log transformations were used for leptin and total adiponectin, because they are not normally distributed. Multivariate-adjusted regression coefficients are reported associated with a one standard deviation increase in leptin, resistin and adiponectin. All analyses were performed using SAS version 9.2 statistical software (Cary, NG).

\section{Results}

General characteristics of study participants by CKD status are presented in Table 1. Those with CKD were older, less educated, heavier, and less likely to drink alcohol compared to those without CKD. In addition, they were more likely to have a history of CVD, hypertension, diabetes, and hypercholesterolemia and use lipid lowering, anti-diabetes and antihypertensive medications, as well as aspirin. Systolic BP, plasma glucose, and urinary albumin were significantly higher, while LDL-cholesterol, HDL-cholesterol, and eGFR were lower in CKD patients compared to controls.

Median leptin is significantly higher in cases compared to controls $(22.4 \mathrm{ng} / \mathrm{mL}$ vs. $11.6 \mathrm{ng} / \mathrm{mL} ; \mathrm{p}<0.0001)$, and remained significantly higher after full adjustment (Table 2). Mean resistin was also higher in cases than in controls, both adjusted and unadjusted $(16.2$ vs. $8.8 \mathrm{ng} / \mathrm{mL} ; \mathrm{p}<0.0001)$. No significant difference was observed in either unadjusted or fully adjusted median total adiponectin between CKD cases and controls.

In logistic regression analyses adjusted for age, gender and race, those in the highest tertile of leptin and resistin are significantly more likely to have CKD compared to the lower two tertiles (Table 3). The highest tertile of total adiponectin has a nonstatistically significant increased risk of CKD compared to the two lower tertiles. Additional adjustment for high school education, current cigarette smoking, weekly alcohol consumption, physical activity, LDL-cholesterol, systolic BP, and serum glucose did not substantially change the associations between leptin, resistin, and adiponectin and CKD. After further adjustment for BMI, the highest tertiles of leptin, resistin, and adiponectin were significantly associated with CKD compared to the lower two tertiles. Adjusting for waist circumference instead of BMI did not substantially change the associations (results not shown). In a final model with 
Table 1. Characteristics of 201 patients with chronic kidney disease and 201 controls.

\begin{tabular}{|c|c|c|c|}
\hline & CKD patients & Non-CKD controls & \\
\hline Variables & $(n=201)$ & $(n=201)$ & $p$-value for difference \\
\hline Age, years & $55.9 \pm 9.9$ & $52.5 \pm 10.0$ & 0.0007 \\
\hline Male, \% & 55.2 & 45.3 & 0.05 \\
\hline Blacks, \% & 60.7 & 51.2 & 0.06 \\
\hline High school education, \% & 58.5 & 81.6 & $<0.0001$ \\
\hline Current cigarette smoking, \% & 53.7 & 48.8 & 0.32 \\
\hline Weekly alcohol drinking, \% & 27.9 & 59.2 & $<0.0001$ \\
\hline Regular physical activity*, \% & 53.0 & 72.9 & $<0.0001$ \\
\hline History of CVD, \% & 43.7 & 7.0 & $<0.0001$ \\
\hline History of hypertension, \% & 88.1 & 23.9 & $<0.0001$ \\
\hline History of diabetes, \% & 49.3 & 5.5 & $<0.0001$ \\
\hline History of hypercholesterolemia, \% & 65.7 & 30.9 & $<0.0001$ \\
\hline Lipid-lowering Medication Use, \% & 23.0 & 9.0 & 0.0001 \\
\hline Anti-diabetic Medication Use, \% & 37.2 & 3.0 & $<0.0001$ \\
\hline Antihypertensive Medication Use, \% & 82.7 & 15.4 & $<0.0001$ \\
\hline Aspirin Use, \% & 35.6 & 8.5 & $<0.0001$ \\
\hline Body-mass index, $\mathrm{kg} / \mathrm{m}^{2}$ & $32.2 \pm 7.8$ & $28.9 \pm 6.4$ & $<0.0001$ \\
\hline Systolic blood pressure, $\mathrm{mmHg}$ & $132.2 \pm 21.0$ & $122.0 \pm 14.7$ & $<0.0001$ \\
\hline Diastolic blood pressure, $\mathrm{mmHg}$ & $77.2 \pm 13.5$ & $77.6 \pm 9.4$ & 0.77 \\
\hline Plasma glucose, mg/dL & $119.9 \pm 46.7$ & $103.4 \pm 35.4$ & $<0.0001$ \\
\hline LDL-cholesterol, mg/dL & $103.3 \pm 45.9$ & $118.2 \pm 30.2$ & $<0.0001$ \\
\hline HDL-cholesterol, mg/dL & $50.3 \pm 15.6$ & $57.7 \pm 18.0$ & $<0.0001$ \\
\hline eGFR, mL/min/1.73 m² & $43.3 \pm 19.9$ & $96.7 \pm 16.8$ & $<0.0001$ \\
\hline Urinary albumin, mg/24-hrs & $74.5(12.3,417.4)$ & $5.9(4.1,11.4)$ & $<0.0001$ \\
\hline
\end{tabular}

additional adjustment for history of CVD, the odds of CKD in the highest tertiles of leptin, resistin, and adiponectin remained significantly higher than the odds in the lower two tertiles.

Multivariate-adjusted linear regression of leptin, resistin, and adiponectin with eGFR and urinary albumin showed a significant inverse association with eGFR and a significant positive association with albumin and each of the adipocytokines (Table 4). The direction of these associations is consistent with leptin, resistin, and adiponection being positively associated with CKD severity.

Table 2. Adipocytokine levels according to chronic kidney disease status.

\begin{tabular}{|c|c|c|c|c|c|c|}
\hline & \multicolumn{3}{|c|}{ Unadjusted median (IQR) } & \multicolumn{3}{|c|}{ Multivariable-adjusted median (IQR)* } \\
\hline & CKD patients & Non-CKD controls & & CKD patients & Non-CKD controls & \\
\hline Adipocytokines & $(n=201)$ & $(n=201)$ & $p$-value for difference & $(n=201)$ & $(n=201)$ & $\mathrm{p}$-value for difference \\
\hline \multirow[t]{2}{*}{ Leptin (ng/mL) } & 22.4 & 11.6 & $<0.0001$ & 38.2 & 17.2 & $<0.0001$ \\
\hline & $(10.8,58.9)$ & $(4.7,25.0)$ & & $(24.1,56.4)$ & $(11.7,24.5)$ & \\
\hline \multirow[t]{2}{*}{ Resistin $(\mathrm{ng} / \mathrm{mL})^{\dagger}$} & 16.2 & 8.8 & $<0.0001$ & 16.2 & 9.0 & $<0.0001$ \\
\hline & $(15.1,17.4)$ & $(8.2,9.5)$ & & $(15.2,17.2)$ & $(7.9,10.0)$ & \\
\hline \multirow[t]{2}{*}{ Total Adiponectin $(\mu \mathrm{g} / \mathrm{mL})$} & 5.2 & 5.1 & 0.5 & 5.9 & 5.5 & 0.1 \\
\hline & $(3.5,9.4)$ & $(3.4,8.4)$ & & $(3.7,10.1)$ & $(4.3,7.7)$ & \\
\hline
\end{tabular}

$\mathrm{IQR}=$ inter-quartile range.

*Adjusted for age, gender, race, high school education, current cigarette smoking, weekly alcohol consumption, physical activity, body-mass index, LDL-cholesterol, systolic blood pressure, serum glucose, and history of cardiovascular disease.

${ }^{\dagger}$ Mean (95\% confidence interval).

doi:10.1371/journal.pone.0076902.t002 
Table 3. Odds ratios of chronic kidney disease associated with highest tertile compared to the lowest two tertiles adipocytokines.

\begin{tabular}{|c|c|c|c|c|c|c|c|c|}
\hline & \multicolumn{2}{|c|}{ Age, gender, and race-adjusted } & \multicolumn{2}{|c|}{ Multivariable-adjusted ${ }^{*}$} & \multicolumn{2}{|c|}{ Multivariable-adjusted $^{* *}$} & \multicolumn{2}{|c|}{ Multivariable-adjusted $^{\dagger}$} \\
\hline & OR $(95 \% \mathrm{Cl})$ & $\mathrm{p}$-value & OR (95\% Cl) & $\mathrm{p}$-value & OR $(95 \% \mathrm{Cl})$ & $\mathrm{p}$-value & OR $(95 \% \mathrm{Cl})$ & $\mathrm{p}$-value \\
\hline \multicolumn{9}{|c|}{ Leptin (ng/mL) } \\
\hline$<20.35$ & 1.0 (ref) & $<0.0001$ & 1.0 (ref) & 0.0002 & 1.0 (ref) & 0.006 & 1.0 (ref) & 0.03 \\
\hline$\geq 20.35$ & $3.89(2.29,6.60)$ & & $3.20(1.73,5.91)$ & & $2.74(1.34,5.61)$ & & $2.32(1.09,4.94)$ & \\
\hline \multicolumn{9}{|c|}{ Resistin (ng/mL) } \\
\hline$<9.42$ & 1.0 (ref) & $<0.0001$ & 1.0 (ref) & $<0.0001$ & 1.0 (ref) & $<0.0001$ & 1.0 (ref) & $<0.0001$ \\
\hline$\geq 9.42$ & $11.2(6.8,18.6)$ & & $10.5(5.9,18.7)$ & & $11.0(6.1,20.0)$ & & $12.7(6.5,24.6)$ & \\
\hline \multicolumn{9}{|c|}{ Total Adiponectin $(\mu \mathrm{g} / \mathrm{mL})$} \\
\hline$<7.24$ & 1.0 (ref) & 0.1 & 1.0 (ref) & 0.06 & 1.0 (ref) & 0.02 & 1.0 (ref) & 0.03 \\
\hline$\geq 7.24$ & $1.44(0.91,2.30)$ & & $1.70(0.98,2.95)$ & & $2.03(1.14,3.61)$ & & $1.95(1.06,3.56)$ & \\
\hline
\end{tabular}

*Adjusted for age, gender, race, high school education, current cigarette smoking, weekly alcohol consumption, physical activity, LDL-cholesterol, systolic blood pressure, and serum glucose.

${ }^{* *}$ Additionally adjusted for body mass index.

${ }^{\dagger}$ Additionally adjusted for history of cardiovascular disease.

doi:10.1371/journal.pone.0076902.t003

\section{Discussion}

The results of the current study show that plasma leptin and resistin concentrations are significantly higher in patients with CKD compared to those without, while there was no significant difference in median total adiponectin between CKD patients and controls. There was a significant and independent association of elevated levels of leptin, resistin, and adiponectin with odds of CKD. In addition, leptin, resistin, and adiponectin are significantly positively associated with severity of CKD measured by eGFR and urinary albumin. The observed associations are independent of established risk factors for CKD, including systolic blood pressure, serum glucose, history of cardiovascular disease, and body mass index.

Our study has important clinical implications. Our findings provide further evidence that leptin and resistin are significantly higher in CKD compared to non-CKD participants. In addition, our results show that leptin, resistin and adiponectin are not only associated with the risk, but also the severity of CKD, independent of BMI and GVD.

Our positive findings of leptin and resistin with CKD are consistent with prior studies [12-14,16-18,25]. Several studies have found significantly higher levels of leptin and resistin in those with CKD compared to healthy controls with minimal or no multivariate adjustment $[12,13,16,17,25]$. A recent analysis by Shankar et al using data from the Third National Health and
Nutrition Examination Survey of 5,820 representative adults in the United States found the odds of CKD to be 3.25 (95\% CI: 1.61, 6.55) times higher in the highest quartile of leptin compared to the lowest quartile after adjusting for relevant covariates [14]. A study of 3,192 Japanese subjects found an odds ratio for CKD of 2.32 (95\% CI: $1.71,3.16)$ comparing those in the highest resistin tertile to the lowest [18]. Our case-control study confirms these previous findings after adjustment for relevant covariates and has the additional strength of using proteinuria to define CKD, which was not a part of the case definition in previous multivariate analyses and allows for a more accurate classification of CKD cases and non-cases.

Adiponectin has anti-inflammatory properties [26,27] and is inversely associate with obesity, coronary artery disease, and type 2 diabetes [26,28,29]. Contrary to these findings, prior studies of adiponectin and CKD have shown either higher adiponectin levels in those with CKD compared to those without CKD $(19.2 \mu \mathrm{g} / \mathrm{mL}$ vs $14.8 \mu \mathrm{g} / \mathrm{mL} ; \mathrm{p}<0.01)$ [19] or no difference in adiponectin levels between the two groups in unadjusted analyses $[13,17,21]$. While we observed no difference between the median values of adiponectin between cases and controls, those with higher adiponectin values have greater odds of GKD in our study, and the association remained significant after multivariate adjustment. In addition, we found a significant positive association between adiponectin and markers of disease severity consistent with some prior studies [30-31].

Table 4. Multivariable-adjusted regression coefficients ( $95 \%$ confidence intervals) of GFR and urinary albumin associated with a one standard deviation difference in adipocytokines.

\begin{tabular}{|c|c|c|c|c|}
\hline & \multicolumn{2}{|c|}{ eGFR, $\mathrm{mL} / \mathrm{min} / 1.73 \mathrm{~m}^{2}$} & \multicolumn{2}{|c|}{ Log (urinary albumin, mg/24-hrs) } \\
\hline & $\beta(95 \% \mathrm{Cl})$ & p-value & $\beta(95 \% \mathrm{Cl})$ & p-value \\
\hline Log (leptin, 1.25 ng/mL) & $-12.7(-17.4,-7.9)$ & $<0.0001$ & $0.64(0.34,0.94)$ & $<0.0001$ \\
\hline Resistin $(7.58$ ng/mL) & $-15.0(-17.7,-12.4)$ & $<0.0001$ & $0.74(0.56,0.91)$ & $<0.0001$ \\
\hline Log (total adiponectin, $0.66 \mu \mathrm{g} / \mathrm{mL}$ ) & $-8.7(-11.7,-5.6)$ & $<0.0001$ & $0.36(0.16,0.57)$ & 0.0004 \\
\hline
\end{tabular}

eGFR = estimated-glomerular filtration rate.

*Adjusted for age, gender, race, high school education, current cigarette smoking, weekly alcohol consumption, physical activity, body-mass index, LDL-cholesterol, systolic blood pressure, serum glucose, and history of cardiovascular disease.

doi:10.1371/journal.pone.0076902.t004 
This study has several strengths and limitations. First, these analyses had a larger sample size than most previous studies of these associations. Second, these analyses adjusted for many CKD risk factors, so the independent association between adipocytokines and CKD could be assessed. Third, proteinuria was measured in this population and used to define CKD allowing for a more accurate classification of case status than eGFR alone. The cross-sectional nature of this study does not allow us to determine if the higher levels of adipocytokines lead to kidney damage and CKD or if the higher levels are a result of kidney damage associated with CKD. Longitudinal studies are needed to further understand these associations.

In conclusion, our results show that leptin, resistin, and adiponectin are associated with the risk and severity of CKD. These results suggest that longitudinal studies and clinical trials

\section{References}

1. Coresh J, Selvin E, Stevens LA, Manzi J, Kusek JW, et al. (2007) Prevalence of chronic kidney disease in the united states. JAMA 298: 2038-2047.

2. Levey AS, Coresh J (2012) Chronic kidney disease. Lancet 379: 165-180.

3. Go AS, Chertow GM, Fan D, McCulloch CE, Hsu CY (2004) Chronic kidney disease and the risks of death, cardiovascular events, and hospitalization. N Engl J Med 351: 1296-1305.

4. Gerstein HC, Mann JF, Yi Q, Zinman B, Dinneen SF, et al. (2001) Albuminuria and risk of cardiovascular events, death, and heart failure in diabetic and nondiabetic individuals. JAMA 286: 421-426.

5. Chronic Kidney Disease Prognosis Consortium, Matsushita K, van der Velde M, Astor BC, Woodward M, et al. (2010) Association of estimated glomerular filtration rate and albuminuria with all-cause and cardiovascular mortality in general population cohorts: A collaborative meta-analysis. Lancet 375: 20732081.

6. Fox CS, Larson MG, Leip EP, Culleton B, Wilson PW, et al. (2004) Predictors of new-onset kidney disease in a community-based population. JAMA 291: 844 850.

7. Schiffrin EL, Lipman ML, Mann JF (2007) Chronic kidney disease: Effects on the cardiovascular system. Circulation 116: 85-97.

8. Ntaios G, Gatselis NK, Makaritsis K, Dalekos GN (2013) Adipokines as mediators of endothelial function and atherosclerosis. Atherosclerosis 227: 216221.

9. Merabet E, Dagogo-Jack S, Coyne DW, Klein S, Santiago JV, et al. (1997) Increased plasma leptin concentration in end-stage renal disease. J Clin Endocrinol Metab 82: 847-850.

10. Sharma K, Considine RV, Michael B, Dunn SR, Weisberg LS, et al. (1997) Plasma leptin is partly cleared by the kidney and is elevated in hemodialysis patients. Kidney Int 51: 1980-1985.

11. Nordfors L, Lonnqvist F, Heimburger O, Danielsson A, Schalling M, et al. (1998) Low leptin gene expression and hyperleptinemia in chronic renal failure. Kidney Int 54: 1267-1275.

12. Fruehwald-Schultes B, Kern W, Beyer J, Forst T, Pfutzner A, et al. (1999) Elevated serum leptin concentrations in type 2 diabetic patients with microalbuminuria and macroalbuminuria. Metabolism 48: 1290-1293.

13. Guebre-Egziabher F, Bernhard J, Funahashi T, Hadj-Aissa A, Fouque D (2005) Adiponectin in chronic kidney disease is related more to metabolic disturbances than to decline in renal function. Nephrol Dial Transplant 20: 129-134.

14. Shankar A, Syamala S, Xiao J, Muntner P (2012) Relationship between plasma leptin level and chronic kidney disease. Int J Nephrol 2012: 269532.

15. de Vinuesa SG, Goicoechea M, Kanter J, Puerta M, Cachofeiro V, et al. (2006) Insulin resistance, inflammatory biomarkers, and adipokines in patients with chronic kidney disease: Effects of angiotensin II blockade. J Am Soc Nephrol 17: S206-12.

16. Axelsson J, Bergsten A, Qureshi AR, Heimburger O, Barany P, et al. (2006) Elevated resistin levels in chronic kidney disease are associated with decreased glomerular filtration rate and inflammation, but not with insulin resistance. Kidney Int 69: 596-604. should be conducted to investigate if adipocytokines play a role in the development and progression of CKD independent of BMI or waist circumference. These important findings may advance our further understanding of risk factors for CKD.

\section{Acknowledgments}

The authors would like to express their appreciation to all study staff and participants for their assistance in this project.

\section{Author Contributions}

Conceived and designed the experiments: JC JH. Performed the experiments: LLH ABA CM AH SB JT MK JC. Analyzed the data: KM CSC YL. Contributed reagents/materials/analysis tools: NR. Wrote the paper: KM JH JC.

17. Yaturu S, Reddy RD, Rains J, Jain SK (2007) Plasma and urine levels of resistin and adiponectin in chronic kidney disease. Cytokine 37: 1-5.

18. Kawamura R, Doi Y, Osawa H, Ninomiya T, Hata J, et al. (2010) Circulating resistin is increased with decreasing renal function in a general japanese population: The hisayama study. Nephrol Dial Transplant 25: 3236-3240.

19. Norata GD, Baragetti I, Raselli S, Stucchi A, Garlaschelli K, et al. (2010) Plasma adiponectin levels in chronic kidney disease patients: Relation with molecular inflammatory profile and metabolic status. Nutr Metab Cardiovasc Dis 20: 5663

20. Zoccali C, Mallamaci F, Tripepi G, Benedetto FA, Cutrupi S, et al. (2002) Adiponectin, metabolic risk factors, and cardiovascular events among patients with end-stage renal disease. J Am Soc Nephrol 13: 134-141.

21. Becker B, Kronenberg F, Kielstein JT, Haller H, Morath C, et al. (2005) Renal insulin resistance syndrome, adiponectin and cardiovascular events in patients with kidney disease: The mild and moderate kidney disease study. J Am Soc Nephrol 16: 1091-1098.

22. Perloff D, Grim C, Flack J, Frohlich ED, Hill M, et al. (1993) Human blood pressure determination by sphygmomanometry. Circulation 88: 2460-2470.

23. Levey AS, Stevens LA, Schmid CH, Zhang YL, Castro AF 3rd, et al. (2009) A new equation to estimate glomerular filtration rate. Ann Intern Med 150: 604 612 .

24. Hsieh CC, Maisonneuve P, Boyle P, Macfarlane GJ, Roberston G (1991) Analysis of quantitative data by quantiles in epidemiologic studies: Classification according to cases, noncases, or all subjects? Epidemiology 2: 137-140.

25. Sarnak MJ, Poindexter A, Wang SR, Beck GJ, KusekJW, et al. (2002) Serum Creactive protein and leptin as predictors of kidney disease progression in the modification of diet in renal disease study. Kidney Int 62: 2208-2215.

26. Ouchi N, Kihara S, Arita Y, Maeda K, Kuriyama H, et al. (1999) Novel modulator for endothelial adhesion molecules: Adipocyte-derived plasma protein adiponectin. Circulation 100: 2473-2476.

27. Ouchi N, Kihara S, Arita Y, Okamoto Y, Maeda K, et al. (2000) Adiponectin, an adipocyte-derived plasma protein, inhibits endothelial NF-kappaB signaling through a cAMP-dependent pathway. Circulation 102: 1296-1301.

28. Hotta K, Funahashi T, Arita Y, Takahashi M, Matsuda M, et al. (2000) Plasma concentrations of a novel, adipose-specific protein, adiponectin, in type 2 diabetic patients. Arterioscler Thromb Vasc Biol 20: 1595-1599.

29. Weyer C, Funahashi T, Tanaka S, Hotta K, Matsuzawa Y, et al. (2001) Hypoadiponectinemia in obesity and type 2 diabetes: Close association with insulin resistance and hyperinsulinemia. J Clin Endocrinol Metab 86: 19301935.

30. Nanayakkara PW, Le Poole CY, Fouque D, van Guldener C, Stehouwer CD, et al. (2009) Plasma adiponectin concentration has an inverse and a non linear association with estimated glomerular filtration rate in patients with K/DOQI 3 - 5 chronic kidney disease. Clin Nephrol 72: 21-30.

31. Kollerits B, Fliser D, Heid IM, Ritz E, Kronenberg F, et al. (2007) Genderspecific association of adiponectin as a predictor of progression of chronic kidney disease: The mild to moderate kidney disease study. Kidney Int 71: 1279-1286. 